\title{
High Ureteric Injury Following Multiorgan Recovery: Successful Kidney Transplant with Boari Flap Ureterocystostomy Reconstruction
}

\author{
Michael Charlesworth*, Gabriele Marangoni, Niaz Ahmad \\ Department of Transplantation, Division of Surgery, St James's University Hospital, Leeds, United Kingdom
}

\begin{abstract}
Introduction: Despite increased utilization of marginal organs, there is still a marked disparity between organ supply and demand for transplantation. To maximize resources, it is imperative that procured organs are in good condition. Surgical damage at organ recovery can happen and organs are sometimes discarded as a result. We describe a damaged recovered kidney with high ureteric transection that was successfully transplanted using a primary Boari flap ureterocystostomy.
\end{abstract}

Case report: The donor kidney was procured form a deceased donor and sustained damage by transection of the ureter just distal to the pelvi-ureteric junction at organ recovery. The recipient had been on the transplant waiting list for eight years and not accepting this kidney would have seriously jeopardized her chance of future transplantation. Several centers rejected the donor kidney. We assessed the kidney and decided to proceed with transplantation. The kidney was successfully transplanted. A primary Boari flap was constructed and the transplant ureter implanted onto the bladder. The post-operative course was complicated by urine leak that settled down after management by nephrostomy insertion and bladder catheterization. The patient made a good recovery and remained well at six monthly follow up with a serum creatinine of $1.7 \mathrm{mg} / \mathrm{dl}$.

Conclusion: Primary Boari flap ureterocystostomy is a viable option when the transplant surgeon is faced with a high ureteric injury in the procured kidney. Since most patients wait years for a suitable graft and some may never find an adequate match, every effort should be made to effectively utilize the scarce available resources to the fullest.

\footnotetext{
* Corresponding Author; Mr Niaz Ahmad; Consultant Transplant Surgeon, St. James's University Hospital, Beckett Street, Leeds, LS9 7TF, United Kingdom; E mail: niaz.ahmad@leedsth.nhs.uk
}

Keywords: Kidney; Transplant; Ureter; Donor efficiency

\section{The authors declared no conflict of interest}

\section{Introduction}

Despite the extension of the donor pool with the inclusion of marginal organs and the use of organs donated after cardiac death, there is still a great disparity between the number of patients on the transplant waiting list and the number of kidney transplants performed each year. It is therefore of paramount importance to maximize our scarce resources and avoid the discard of otherwise functional kidneys due to iatrogenic injuries at the time of multi-organ recovery. Essentially, three types of organ damage can potentially occur: vascular, parenchymal and ureteric. Should an injury take place, it is crucial that the retrieval team recognizes the damage and that correct communication ensues with the transplant surgeon. The consequences of procurement errors can be minimized with an appropriate surgical strategy so that the kidney could still be transplanted successfully. We describe a case of high ureteric injury during multi-organ recovery and successful implantation using a Boari flap ureterocystostomy.

\section{Case Report}

The donor kidney was procured from a 55-year-old male brain stem deceased donor following intra-cerebral hemorrhage. The kidney was damaged by transection of the ureter just distal to the pelvi-ureteric junction at organ recovery (Figure-1). The recipient was a 63-yearold lady whom had been on the transplant waiting list for 8 years. There had been much difficulty during this period to find a suitably matched kidney. Non-acceptance of this kidney would have seriously jeopardized the chance of future transplantation for this lady. Several centers rejected the donor kidney due to the damage caused at organ recovery. The kidney was assessed at 
Figure 1: Preoperative image demonstrating transection of the ureter distal to the pelvic-ureteric junction of the donor kidney

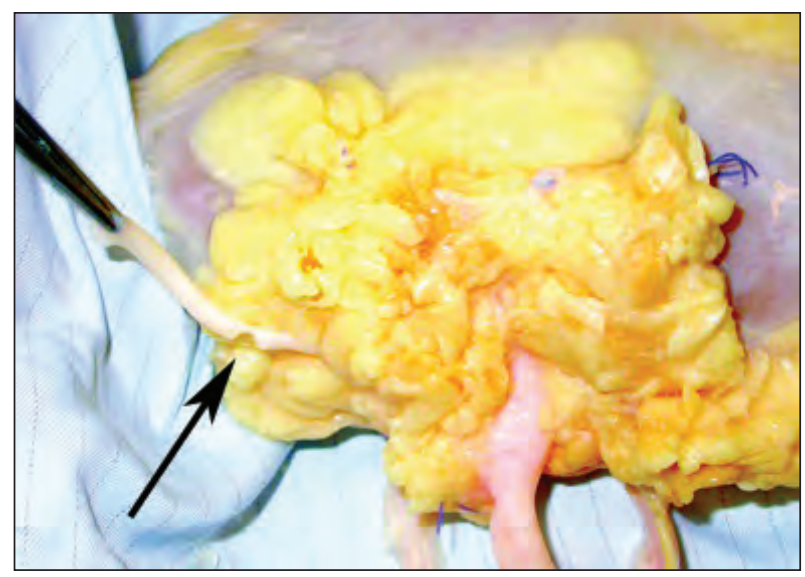

Figure 2: Peri-operative imaging demonstrating Boari flap construction following successful implantation onto the bladder

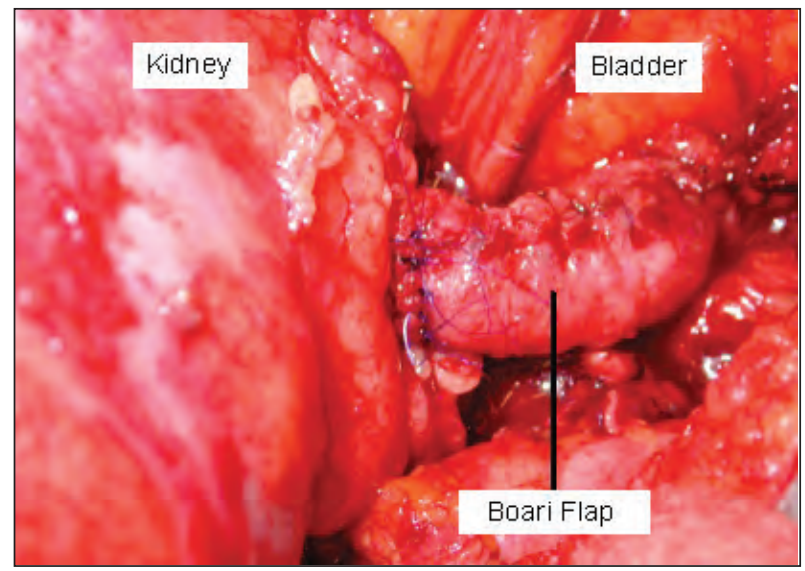

Figure 3: A schematic of a Boari flap uretocystostomy as depicted in the operation sheet

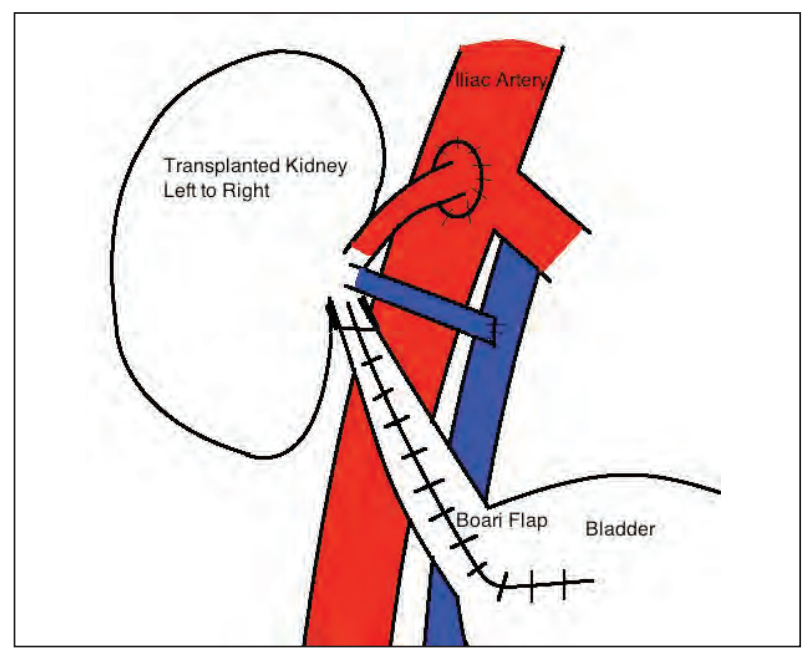

our center and a decision was undertaken to proceed with transplantation. A standard retroperitoneal approach was employed for transplantation of the kidney with vascular anastamosis to the two iliac vessels. The options of using the native ureter or a psoas hitch procedure were considered, but deemed inappropriate for this case. A primary Boari flap was constructed and the transplant ureter implanted onto the bladder. A peri-operative image of the successfully transplanted kidney with Boari flap construction is displayed following implantation in Figure-2. A schematic of the operation note diagram is demonstrated in Figure-3.

The post-operative course was complicated by bleeding resulting in surgical re-exploration and subsequently by urine leak. This was managed by nephrostomy insertion and bladder catheterization. A urogram via the nephrostomy demonstrated a small leak from the Boari flap. This settled without the need for further intervention. Renal function improved over time and the serum creatinine stabilized at $1.8 \mathrm{mg} / \mathrm{dl}$. An ultrasound scan and a cystogram prior to nephrostomy removal did not demonstrate hydronephrosis or a leak. The patient was discharged on post-operative day 58 . At six monthly follow up, the patient remained well with good urine output and stable serum creatinine at $1.7 \mathrm{mg} / \mathrm{dl}$.

\section{Discussion}

The published literature regarding damaged organs for transplantation is scarce. Donated organs are a precious commodity. High standards of organ recovery are a necessity to ascertain all organs recovered are suitable for transplantation. The Human Organ Transplants Act 1984 [1] requires the name of the surgeon responsible for organ recovery to be documented in addition to any damage to recovered organs on a standard form. It is widely perceived that surgical damage during organ retrieval is under reported. In extreme cases of damage, organs are obviously unsuitable and discarded. In lesser cases, the decision is seldom clear and often subjective based on the description of the retrieving surgeon and without assessment of the surgeon who declines the kidney.

Wigmore et al reported the incidence of kidney damage during retrieval in the UK [2]. Their report spanned the period 1992-1996 in which 9014 kidneys were recovered from cadaveric donors. Of these, 1726 (19.1\%) were reported as damaged and 1630 were still transplanted with $96(1.1 \%)$ being discarded. The trends of damage and transplant remained unchanged during this 5-year period, regardless of the zonal kidney transplantation system established in November 1993. It is important to note that the vast majority of damaged kidneys were 
still transplanted [2]. The lowest proportion of damaged kidneys occurred when the liver team was involved and in centers with more than 50 retrievals per year. This implies that a wider dissection during organ procurement and multi-organ retrieval units encompassing large populations may decrease the risk of organ procurement damage. Fernandez et al report a loss of 2 out of 266 retrieved kidneys for vascular damage $(0.75 \%)$ with a high rate of vascular $(16 \%)$ and ureteric $(10 \%)$ lesions perhaps indicating the need for better procurement standardization [3]. Similarly, Eschwege et al described only four kidneys out of $1239(0.32 \%)$ that had to be discarded due to severe vascular injury [4]. Surgical injuries were associated to kidney only retrieval during donation after cardiac death and when the lead surgeon had less experience. More recently, Bentas et al examined the nature and incidence of renal injuries during organ procurement in the Eurotransplant region from 1996 to 2006 [5]. Of 563 procured kidneys $21.3 \%$ were considered inadequately retrieved and $3 \%$ of the injuries involved the ureter. The discard rate was only $0.2 \%$ with a surgical complication rate of 3.4\%. Primary graft function, 3-year graft survival and long-term graft survival were not influenced by the procurement injury. Although the smaller sample size could not corroborate Wigmore's finding [2], a strong discrepancy in the evaluation of organ quality between procurement teams and transplant surgeons was evident, with the former reporting only $38 \%$ of the injuries.

In our case, several transplant centers rejected the damaged kidney, all without proper assessment. The kidney was destined to be discarded and yet was utilised for successful transplantation, using a technique, which is usually used to repair high ureteric injury or stricture. There were several options available to make use of the damaged kidney. The first was to utilize the native ureter from the ipsilateral diseased kidney. This was deemed to be inappropriate as the patient had been anuric for several years with no investigation of her ureters or lower urinary tract. The risk of using a diseased native ureter was high. A psoas-hitch procedure was considered, however, due to eight years of disuse and atrophy with difficult bladder mobilization, this was also considered to be high risk. Numerous other techniques have been described for replacement of long segments of ureter but seem rather complex, often requiring a full laparotomy and marred with poor outcomes [6, 7]. For these reasons, we decided to proceed with primary Boari flap construction. Most techniques of ureteric reconstruction, following loss or stricture of large segments of ureter, are employed as elective techniques chiefly for native ureters. Some of these techniques are used for reconstructive transplant ureteric strictures including Boari flap ureterocystostomy. The latter is seldom used as a primary procedure or to overcome the loss of the whole length of ureter due to damage at retrieval.

Boari originally described the technique of ureterocystostomy for the management of a tuberculosis related inflammatory ureteral stricture in 1894 [8]. Soon after, Spies and colleagues described the viability of the bladder flap to repair loss of a distal native ureter [9]. The Boari flap is now more commonly utilized to repair ureteral damage during pelvic surgery. It has been described as a repair mechanism for ureteral necrosis following renal transplantation [10]. Total ureteral loss after transplantation with a Boari flap has been described in the literature with good results at follow up [11-13]. In these cases, there was no documented evidence of recurrent urinary tract infections or renal function deterioration.

Del Pizzo and colleagues described 20 patients requiring ureteral reconstruction following renal transplant [14]. In 15 of these patients, a modified Boari flap was employed and the authors report promising results. None had evidence of functional obstruction as measured by diuretic renal scanning. Only one patient had reflux with pelvicaliceal dilatation. The authors concluded that the low incidence of reflux may be an advantage of this technique although vesicoureteral reflux has been reported following Boari flap construction. Bleeding, urine leak and late stricture [15] are also other known complications.

Our group [16] reported successful reconstruction of a completely strictured and surgically inaccessible ureter through a salvage vesicocalycostomy. A Boari flap was anastamosed to the lower pole calyx with a satisfactory outcome. The serum creatnine, in our case, remained stable at 6 monthly follow up at $2.1 \mathrm{mg} / \mathrm{dl}$.

\section{Conclusion}

The use of a Boari flap is not a new technique in renal transplantation. Our case, however, presents a novel application for this; the salvage of a damaged kidney that would have otherwise been rejected for transplantation. This has not been reported elsewhere in the literature to the best of our knowledge. The data regarding organ damage at retrieval is limited and its incidence is probably under-reported. The decision to reject a damaged organ for transplantation is not easily made. Although a rare occurrence, ureteric transection during organ procurement may be repaired using the Boari flap technique. 


\section{References}

2. Wigmore SJ, Seeney FM, Pleass HC, Praseedom RK, Forsythe JL. Kidney damage during organ retrieval: data from UK National Transplant Database. Kidney Advisory Group. Lancet. 1999 Oct 2;354(9185):1143-6.

3. Fernández ED, Schmid M, Schlosser K, Mauer D; Working Group of the Organ Procurement Central Region of the German Foundation for Organ Transplantation (DSO). Technical complications in organ procurement. Transplant Proc. 2007 Dec;39(10):2975-6.

4. Eschwège P, Droupy S, Blanchet P, Hammoudi Y, Laassou K, Hadj AE, Giuliano F, Izard V, Duranteau J, Decaux A, Richard C, Devictor D, Joseph L, Decaris J, Paradis V, Bedossa P, Huault G, Durrbach A, Charpentier B, Benoît G. Surgical injuries occurring during kidney procurement performed by a renal transplantation team. Transplant Proc. 2002 May;34(3):844.

5. Bentas W, Jones J, Urbschat A, Tilp U, Probst M, Scheuermann E, Hauser IA, Blaheta RA, Jonas D, Gossmann J. Effect of procurement-related organ lesions on renal transplant outcome. Clin Transplant. 2008 JulAug;22(4):411-7. Epub 2008 Feb 3.

6. Baum N, Mobley DF, Carlton CE Jr. Ureteral replacements. Urology. 1975 Feb;5(2):165-71.

7. Donovan MG, Barrett DM. Ureteral prostheses. Semin Urol. 1984 Aug;2(3):158-66.

8. Boari A. Chirurgia dell Uretere. Societa Editrice Dante Alighieri. 1894.
9. Spies JW, Johnson CE, Wilson CS, Reconstruction of the ureter by means of bladder flaps. Proc. Soc. Exp. Biol. Med. 1933 Jan;30:425-6

10. Waltzer WC, Frischer Z, Anaise D, Sato K, Rapaport FT. Restoration of urinary tract continuity after necrosis of the pelvis and ureter in a transplanted kidney: the Boari flap. Transplant Proc. 1984 Oct;16(5):1367-71.

11. Toguri AG, Emtage JB, Jarzylo SV. Management of total ureteral loss after kidney transplantation. Can J Surg. 1983 Nov;26(6):498-9.

12. Kinnaert P, Hall M, Janssen F, Vereerstraeten $P$, Toussaint C, Van Geertruyden J. Ureteral stenosis after kidney transplantation: true incidence and long-term followup after surgical correction. J Urol. 1985 Jan;133(1):17-20.

13. Gerridzen RG. Complete ureteral replacement by Boari bladder flap after cadaveric renal transplant. Urology. 1993 Feb;41(2):154-6.

14. del Pizzo JJ, Jacobs SC, Bartlett ST, Sklar GN. The use of bladder for total transplant ureteral reconstruction. J Urol. 1998 Mar;159(3):750-2; discussion 752-3.

15. Thomalla JV, Lingeman JE, Leapman SB, Filo RS. The manifestation and management of late urological complications in renal transplant recipients: use of the urological armamentarium. J Urol. 1985 Nov;134(5):944-8.

16. Gowda BD, Goldsmith P, Ahmad N. Boari flap vesicocalycostomy: a salvage drainage procedure for complete ureteric stricture and pyelocalyceal fistula. Clin Transplant. 2009 Jan-Feb;23(1):129-31. 